Research Article

\title{
Optimal Graphs in the Enhanced Mesh Networks
}

\author{
Muhammad Shahzad Akhtar $\mathbb{D},{ }^{1}$ Muhammad Imran $\left(\mathbb{D},{ }^{2}\right.$ \\ and Syed Ahtsham ul Haq Bokhary $\mathbb{D D}^{1}$ \\ ${ }^{1}$ Center for Advanced Studies in Pure and Applied Mathematics, Bahauddin Zakaria University, Multan, Pakistan \\ ${ }^{2}$ Department of Mathematical Sciences, United Arab Emirates University, P.O. Box 15551 Al Ain, UAE
}

Correspondence should be addressed to Syed Ahtsham ul Haq Bokhary; sihtsham@gmail.com

Received 10 February 2020; Revised 20 March 2020; Accepted 25 March 2020; Published 1 May 2020

Academic Editor: Andrei V. Kelarev

Copyright $\odot 2020$ Muhammad Shahzad Akhtar et al. This is an open access article distributed under the Creative Commons Attribution License, which permits unrestricted use, distribution, and reproduction in any medium, provided the original work is properly cited.

The degree diameter problem explores the biggest graph (in terms of number of nodes) subject to some restrictions on the valency and the diameter of the graph. The restriction on the valency of the graph does not impose any condition on the number of edges (apart from taking the graph simple), so the resulting graph may be thought of as being embedded in the complete graph. In a generality of the said problem, the graph is taken to be embedded in any connected host graph. In this article, host graph is considered as the enhanced mesh network constructed from the grid network. This article provides some exact values for the said problem and also gives some bounds for the optimal graphs.

\section{Introduction}

All graphs discussed in paper are simple, finite, and undirected. The valency (or degree) of a node (or vertex) in the graph $G$ is the number of edges connected with that node in $G$. The maximal valency of the graph $G$ is indicated by $\Delta(G)$. The distance between two nodes $u$ and $v$ of $G$ is the length of the shortest path between them. The distance between a node $x$ and the set $A$ is defined as $d(x, A)=\min _{a \in A} d(x, a)$. For any $A \subseteq V(G)$, let $G-A$ denote the subgraph of $G$ after removing from $G$ all the nodes of $A$ and all the edges incident to at least one node of $A$. The diameter of the graph $G$ is designated by $D$ and is described as the largest distance between any pair of nodes of the graph $G$. For a given graph $G$ and natural numbers $\Delta$ and $D, N_{G}(\Delta, D)$ indicates the number of nodes of largest subgraph of $G$ with given maximal valency $\Delta$ and given diameter $D$.

The topology of a network such as a telecommunications, multiprocessor, or local area network is generally represented by a graph such that stations or processors are represented by vertices (or nodes) and the links or the connection between these networks are represented by edges. There are many important features in the designing of such networks. One of the important aspects is to put limitation on vertex degree and its diameter. These two parameters in networks are interpreted as follows. By the degree of a node, it is meant to have the number of connections attached to that node; on the contrary, the diameter shows the largest number of links that must be required to transmit a message between any two nodes. The natural question that arises in this case is

"What is then the largest number of nodes in a network with a limited degree and diameter?" If we design the network so that there is no directed edge, then this leads to the Degree/Diameter Problem. More formally, we define it as follows.

Find the largest possible number of vertices $N(\Delta, D)$ in a graph of maximum degree $\Delta$ and diameter $D$.

For a thorough survey of the state of the problem, see $[1,2]$. In the Degree Diameter Problem, only restriction that is imposed on the edges is the maximum degree, so there is considerable freedom in placing edges so as to avoid violating the diameter constraint. In this way, the resulting graph may be thought of as being embedded in the complete graph. In this case, the complete graph is acting as the host graph.

A generalization of the Degree Diameter Problem is to consider the graph as embedded in some host graph, not necessarily the complete graph. This problem becomes more interesting when the host graph is considered as a graph 
obtained from some network. The problem was first posed by Dekker et al.[7] in the following form.

Given connected undirected host graph $G$, an upper bound $\Delta$ for the maximum degree, and an upper bound $D$ for the diameter find the largest connected subgraph of maximum degree $\leq \Delta$ and diameter $\leq D$.

In [3-6], the degree diameter problem of honeycomb network, triangular network, oxide network, and silicate network has been explored. In [7], the largest subgraph $N_{G}(\Delta, D)$ has been determined with multidimensional hexagonal grid as the host graph. In [8-15], some extremal properties of graph networks are discussed. Mesh networks can relay messages using either a flooding technique or a routing technique. With routing, the message is propagated along a path by hopping from node to node until it reaches its destination. To ensure that all its paths are available, the network must allow for continuous connections and must reconfigure itself around broken paths, using self-healing algorithms such as Shortest Path Bridging. Self-healing allows a routing-based network to operate when a node breaks down or when a connection becomes unreliable. As a result, the network is typically quite reliable as there is often more than one path between a source and a destination in the network. Although mostly used in wireless situations, this concept can also apply to wired networks and to software interaction. In this work, we have extended this study to the enhanced mesh network.

The Cartesian product $S \square T$ of the graphs $S$ and $T$ is the graph with node set $V(S) \times V(T)$, where two nodes $\left(x_{1}, y_{1}\right)$ and $\left(x_{2}, y_{2}\right)$ are adjacent if and only if either $x_{1}=x_{2}$ and $y_{1} y_{2} \in E(T)$ or $y_{1}=y_{2}$ and $x_{1} x_{2} \in E(S)$.

Let $P_{m}$ and $P_{n}$ are two paths having nodes $m$ and $n$, respectively. The graph of the grid network $P_{m} \square P_{n}$ is obtained by their Cartesian product. The grid graph network $P_{m} \square P_{n}$ has $m n$ nodes and $2 m n-m-n$ edges. The graph of the two-dimensional infinite grid network is denoted by $P_{\infty} \square P_{\infty}$.

Let $G_{m, n}$ be the graph of the enhanced mesh obtained from $P_{m} \square P_{n}$ by replacing its each $4-$ cycle by a wheel $W_{5}$, the hub of the wheel being a new vertex (see Figure 1). In the graph $G_{m, n}$, each 4-cycle of $P_{m} \square P_{n}$ is divided into four triangles where every node lie on some triangle.

Let us define the edges of the wheel $W_{5}$ as follows:

(i) The edge connected to the hub and left-end vertex of upper horizontal edge in $W_{5}$ is called upper-left hub edge

(ii) The edge connected to the hub and right-end vertex of upper horizontal edge in $W_{5}$ is called upper-right hub edge

(iii) The edge connected to the hub and left-end vertex of lower horizontal edge in $W_{5}$ is called lower-left hub edge

(iv) The edge connected to the hub and right-end vertex of lower horizontal edge in $W_{5}$ is called lower-right hub edge

Since $P_{m} \square P_{n}$ has $(m-1)(n-1)$ squares (4-cycles). Therefore, $G_{m, n}$ has $m n+(m-1)(n-1)=2 m n-m-n+1$ nodes. In $G_{m, n}$, the edges adjacent to the hub vertex in each $W_{5}$ are disjoint; therefore, $G_{m, n}$ has $2 m n-m-n+4(m-1)$ $(n-1)=6 m n-5 m-5 n+4$ edges. Furthermore, we define that any two wheel graphs $W_{5}$ in $G$ are said to be adjacent if they share an edge.

\section{Methodology}

In this paper, we have calculated $N_{G}(\Delta, D)$ in the infinite enhanced mesh network. First, we find the induced closed balls $G_{D}$ of diameter $D$ for $\Delta=8$ whose order is $N_{G}(8, D)$ for $D \geq 2$. The order of $G_{D}$ is the upper bound for $N_{G}(\Delta, D)$. We find $N_{G}(\Delta, D)$ for $\Delta \leq 7$ by deleting minimum number of vertices of $G_{D}$. The rest of the paper is ordered as follows. In Section 3, we consider the case for $\Delta=8$; in Section 4, the case for $\Delta=1,2,3,4,5,6,7$ is discussed.

\section{Result and Discussion}

In this section, the results obtained are discussed. The largest subgraphs are obtained for the enhanced mesh networks for given degree and diameter.

3.1. Largest Subgraphs for $\Delta=8$. Let $G$ denote the infinite Enhanced Mesh Network in the Cartesian plane with $\Delta(G)=8$.

Proposition 1. For $D \geq 2$, let the induced subgraph of $G$ of diameter $D$ and maximal degree 8 is denoted by $G_{D}$. Then, for $k \in \mathbb{N}$,

$$
\left|V\left(G_{D}\right)\right|= \begin{cases}(2 k+1)^{2}, & \text { for } D=2 k, \\ 4 k^{2}+8 k+2, & \text { for } D=2 k+1\end{cases}
$$

Proof. For even diameter $D$, let $G_{D}$ be a closed ball having radius $D / 2$ with center as an eight degree node $n$ of $G$ with node set $\left\{x \in V(G): d_{G}(x, n) \leq(\mathrm{D} / 2)\right\}$. To find the nodes of $G_{D}$, we draw horizontal lines on the nodes of $G_{D}$ and count the nodes on these horizontal lines by adding them from top to bottom.

Thus, for $D=2 k, k \in \mathbb{N}$, we have

$$
\begin{aligned}
\left|V\left(G_{D}\right)\right|= & 1+2+3+\cdots+(2 k-2)+(2 k-1)+2 k \\
& +(2 k+1)+2 k+(2 k-1)+(2 k-2)+\cdots+3 \\
& +2+1=(2 k+1)^{2} .
\end{aligned}
$$

Now, for odd diameter $D$, let $G_{D}$ be a closed ball having radius $(D-1) / 2$ and center as the fixed triangle $T$ with node set $\left\{x \in V(G): d_{G}(x, T) \leq((D-1) / 2)\right\}$.

Then, by counting the nodes on horizontal lines, we have for $D=2 k+1, k \in \mathbb{N}$,

$$
\begin{aligned}
\left|V\left(G_{D}\right)\right|= & 2+3+4+\cdots+(2 k-1)+2 k+(2 k+1) \\
& +(2 k+2)+(2 k+1)+2 k+(2 k-1)+\cdots+4 \\
& +3+2=4 k^{2}+8 k+2 .
\end{aligned}
$$




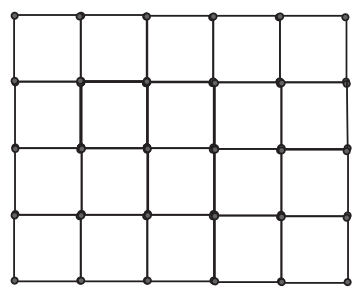

(a)

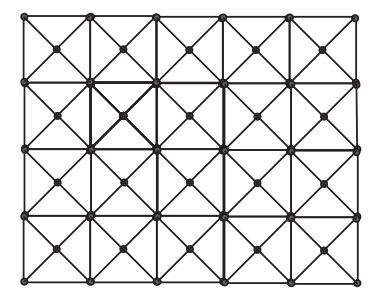

(b)

FIgure 1: Construction of the enhanced mesh from grid graph. (a) $P_{5} \square P_{6}$. (b) $G_{5,6}$.

In Figure 2, the graphs $G_{D}$ for $D=8,9,10$, and 11 are depicted. The central vertex $n$ of $G_{D}$ for even $D$ is depicted by - and for odd diameter $D$, the nodes of central triangle $T$ of $G_{D}$ are indicated by $a, b$, and $c$ and is depicted by $\bullet$. It is important to note that the distance of any node $x$ from $T$ is defined as $d(x, T)=\min _{y \in V(T)} d(x, y)$, where the node $y$ is the nearest node of $T$ from $x$.

Let $L_{D}$ be the biggest connected subgraph of $G$ with maximal degree $\Delta=8$.

Then, for $D=1, L_{D}$ is the triangle $K_{3}$; thus, $N_{G}(8,1)=3$. For $D=2 k+r$, where $k \in \mathbb{N}$ and $r \in\{0,1\}$, the biggest subgraph $L_{D}$ of $G$ with $\Delta=8$ is the closed ball $G_{D}$ itself. Thus, $N_{G}(8, D)=\left|V\left(G_{D}\right)\right|$, for $D=2 k+r, k \in \mathbb{N}, r \in\{0,1\}$.

Hence, we showed the following statement.

Theorem 1. Let $G$ be the graph of enhanced mesh and $D$ be a positive integer. Then,

$N_{G}(8, D)=\left\{\left|V\left(L_{D}\right)\right|=\left|V\left(G_{D}\right)\right|, \quad\right.$ for $D=2 k+r, k \in \mathbb{N}, r \in\{0,1\}$.

Note that the values in the abovementioned theorem are also trivial upper bounds on $N_{G}(\Delta, D)$ for $\Delta \leq 7$. Thus, we prove the following corollary.

Corollary 1. Let $L_{D}$ be the biggest connected subgraph of $G$ of maximal degree 8. Let $\Delta$ and $D$ be positive integers with $\Delta \leq 8$. Then,

$$
N_{G}(\Delta, D) \leq\left|L_{D}\right|
$$

\section{Largest Subgraphs for $\Delta \leq 7$}

We are interested to find the biggest subgraph of $G$ with given maximal valency $\Delta$ and diameter $D$. Since $\Delta(G)=8$, it makes perception to consider the cases for $\Delta \leq 8$. For $\Delta=8$, $N_{G}(\Delta, D)=\left|L_{D}\right|$. Now, we consider the other cases.

4.1. Values for $\Delta=1,2$. If $\Delta=1$, then the biggest connected subgraph of $G$ is just an edge and hence $N_{G}(1, D)=K_{2}$.

Now, we discuss the case when $\Delta=2$.

Theorem 2. Let $G$ be the enhanced mesh network, and let $D$ be a natural number. Then,

$$
N_{G}(2, D)=2 D \text {, when } D \geq 2 \text {. }
$$

Proof. For $D=2 k, k \in \mathbb{N}$. Let $L$ and $M$ be the horizontal and vertical lines, respectively, that are passing through the central node $n$ of $L_{D}$ and divide the graph $L_{D}$ into four regions (upper left, upper right, lower left, and lower right), as shown in Figure 3. Consider a border cycle $C$ of the lowerleft region containing the central node (see the blue cycle in Figure 3(a)). Cycle $C$ has $2 D$ nodes.

For $D=2 k+1, k \in \mathbb{N}$. Let $Q$ be the horizontal line passing through the edge $b c$ and $R$ be the vertical line passing through the node $c$ of the triangle $T$ in the graph $L_{D}$ that divide the graph $L_{D}$ into four regions (upper left, upper right, lower left, and lower right), as shown in Figure 3. Consider a border cycle $C$ of the lower-left region containing the edge $b c$ (see the blue cycle in Figure 3(b)). The cycle $C$ has $2 D$ nodes.

\subsection{Values for $\Delta=7$}

Theorem 3. Let $D$ be an even natural number, and let $G$ be an infinite enhanced mesh network. Then,

$$
\begin{cases}N_{G}(7, D)=8, & \text { for } D=2, \\ N_{G}(7, D)=\left|V\left(L_{D}\right)\right|, & \text { for } D=2 k, k \in \mathbb{N}-\{1\} .\end{cases}
$$

Proof. For $D=2$, the graph contains $K_{1,7}$, which is the biggest subgraph of $G$ of valency 7 , since in the graph $L_{D}$ (shown in Figure 3(a)) of maximal valency $\Delta=8$, the central node can be attached to at most 7 nodes. Hence, $N_{G}(7,2)=8$.

For $D=2 k, k \in\{2,3,4, \ldots\}$, and $\Delta=7$, we construct a subgraph $M_{D}$ (shown in Figure 4) from $L_{D}$ as follows.

Let $L$ be the central horizontal line passing through the central node $n, M$ be the upper neighboring row, and $N$ be the lower neighboring row. Let $H$ be the wheel graph $W_{5}$ of $L_{D}$ lying on and above the central line at a distance zero on the right side of the central node $n$. Furthermore, suppose that $x$ is the hub node of $H$ :

(i) Delete the upper-left and upper-right hub edges in each $W_{5}$ lying on and above the line $L$ of $L_{D}$

(ii) Delete the lower-left and lower-right hub edges in each $W_{5}$ lying on and below the line $L$ of $L_{D}$

(iii) Delete the lower-left hub edge (lower-right hub edge) on left of the central node $n$ (on the right of the central node $n$ ) in each $W_{5}$ lying between the 

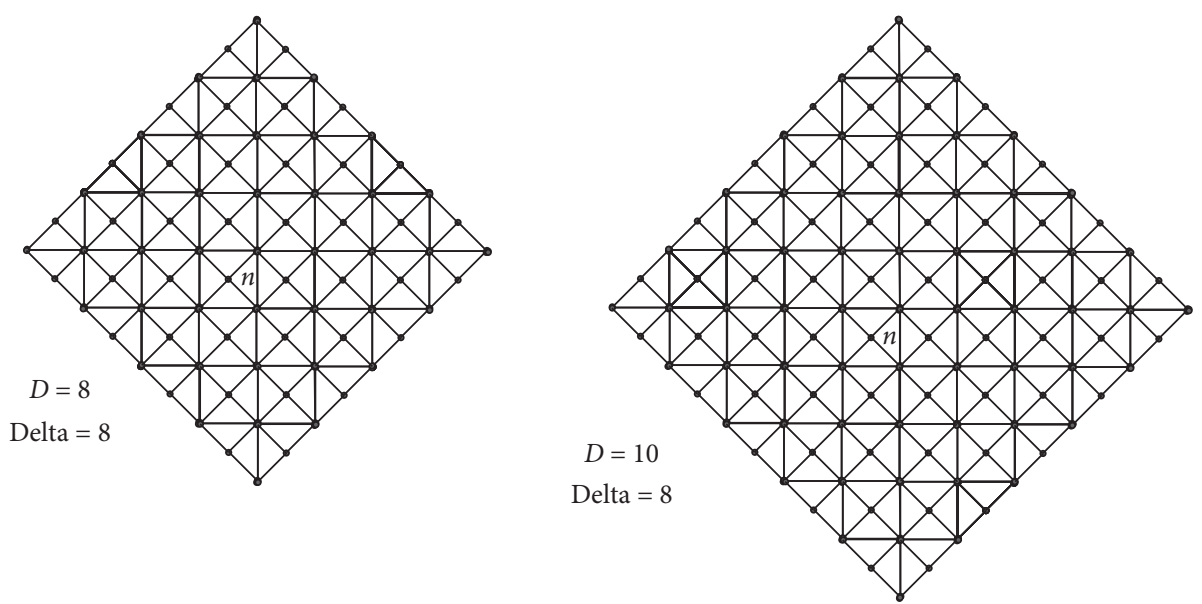

(a)
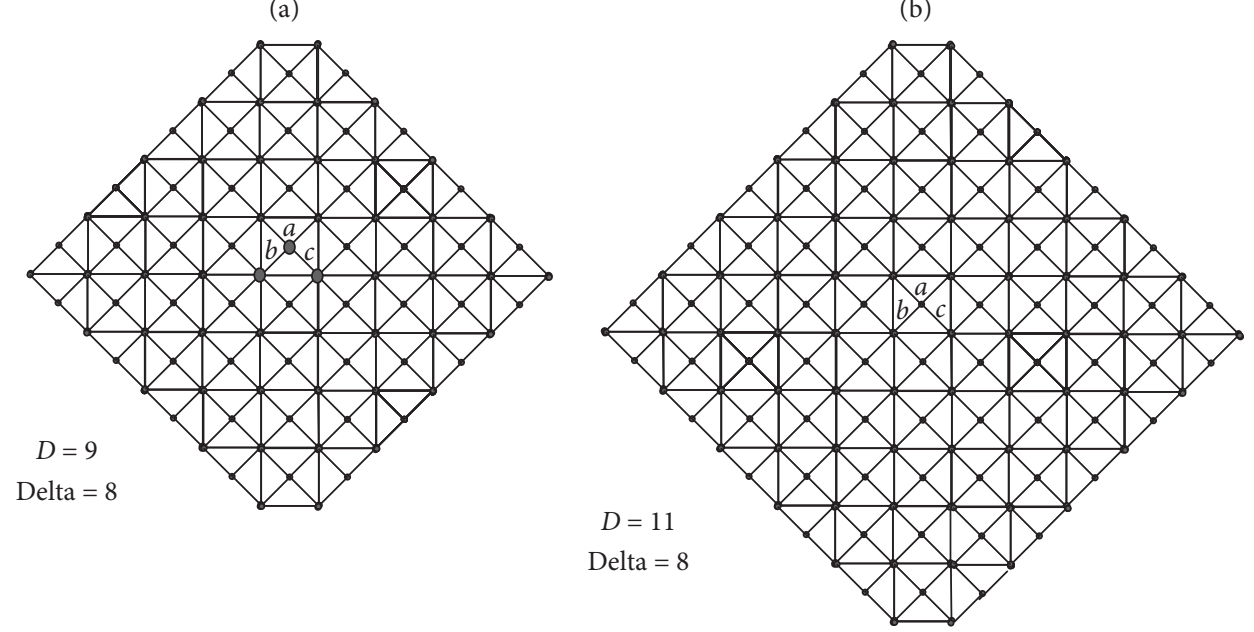

(c)

(d)

FIgURE 2: The graph of $G_{D}$ for $D=8,9,10$, and 11 and $\Delta=8$.
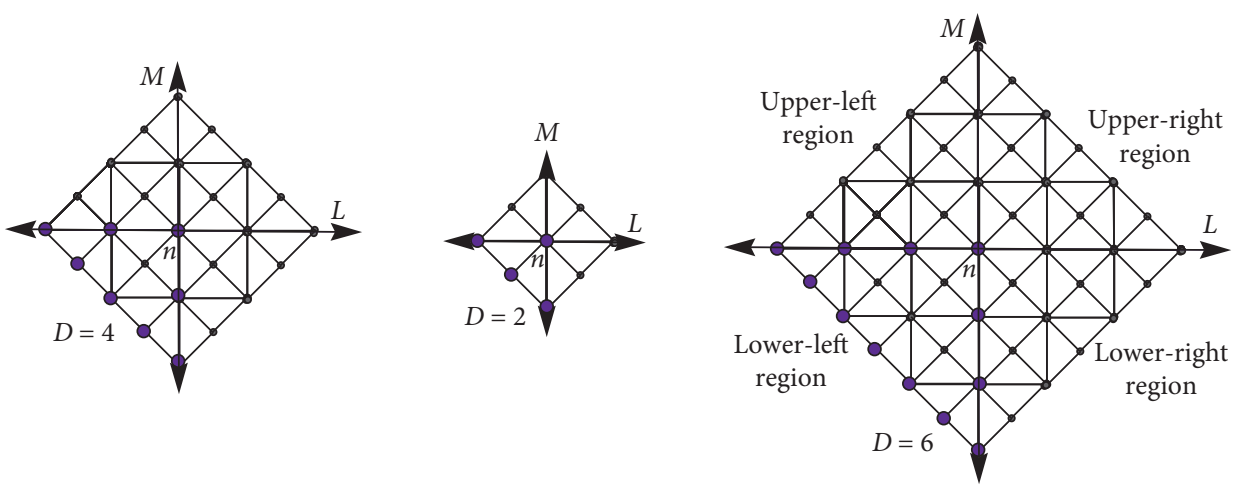

(a)

FIgUre 3: Continued. 

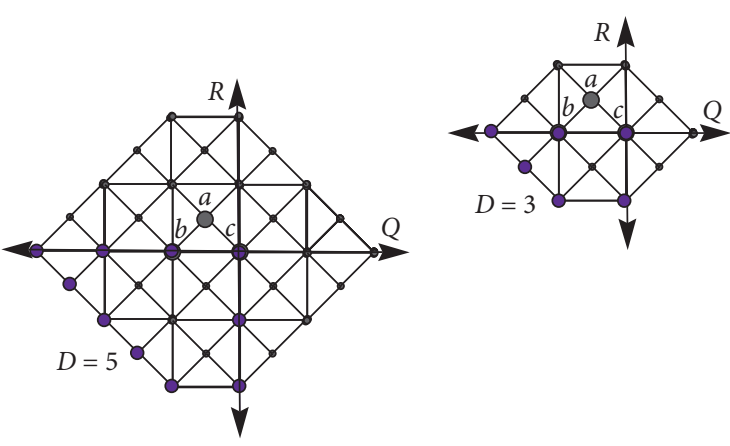

(b)

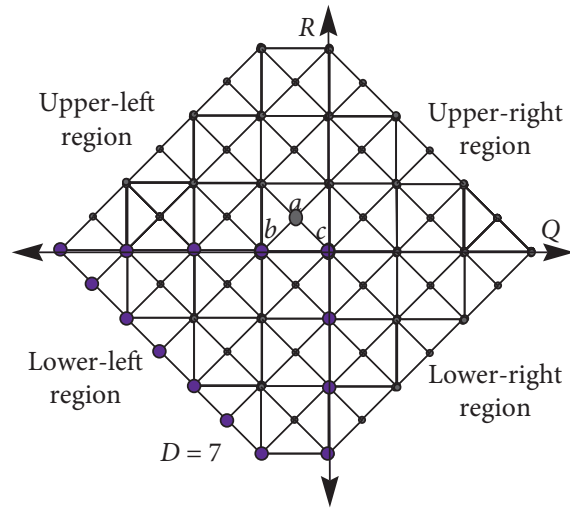

Figure 3: The biggest subgraph $L_{D}$ for $D=2,4,5,6,7$ and $\Delta=2$.
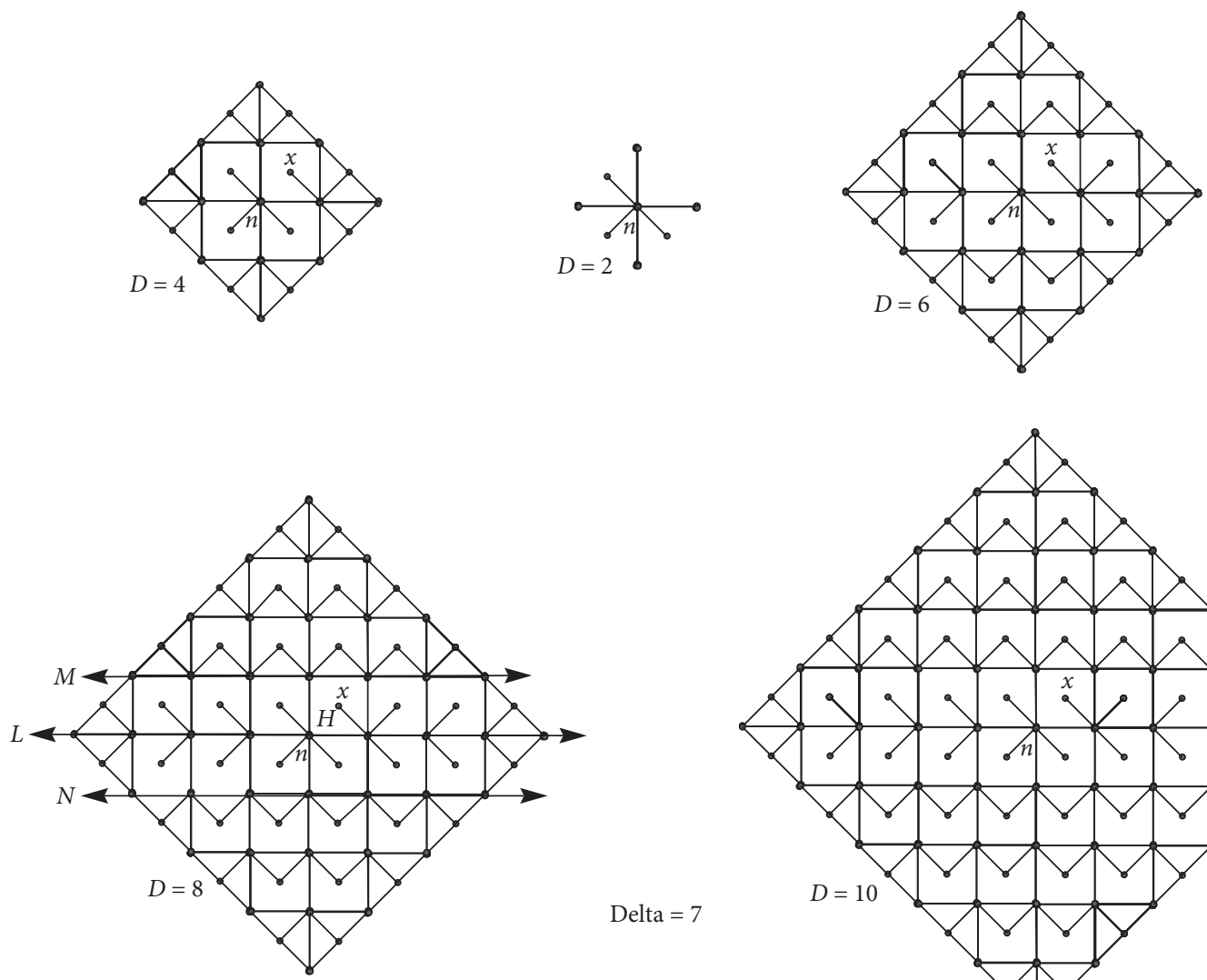

Delta $=7$

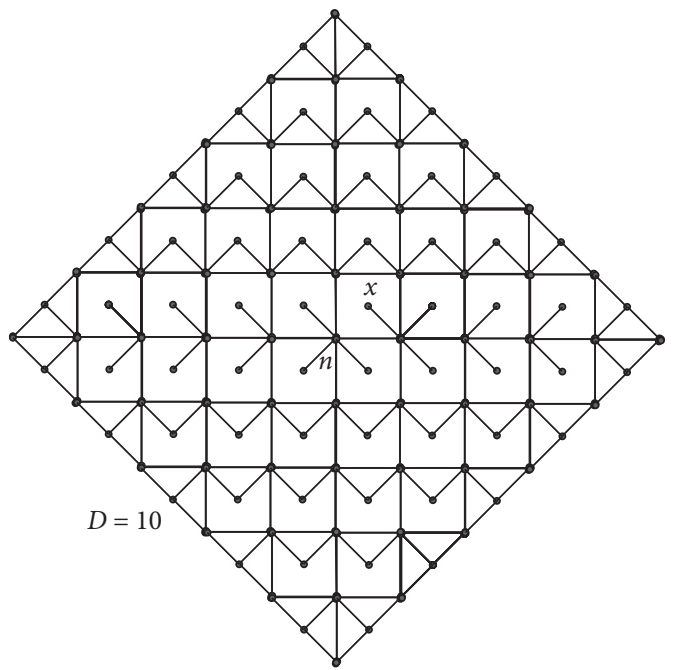

Figure $4: M_{D}$ for $D=4,6,8,10$ and maximal valency $\Delta=7$.

horizontal lines $L$ and $M$ of $L_{D}$, except the wheel graph $H$ whose lower-left hub edge is deleted

(iv) Delete the upper-left hub edge (upper-right hub edge) on left of the central node $n$ (on right of the central node $n$ ) in each $W_{5}$ lying between the lines $L$ and $N$ of the graph $L_{D}$

The resulting subgraph is denoted by $M_{D}$ (shown in Figure 4 , for $D=4,6,8,10$ ) which is the spanning subgraph of $L_{D}$; hence, $V\left(L_{D}\right)=V\left(M_{D}\right)$. Furthermore, graph $M_{D}$ also has diameter $D$ since the distance of all the nodes of $M_{D}$ from $n$ is the same as in $L_{D}$ except the node $x$. However, $d(x, n)=2 \leq(D / 2)$, for all $D \geq 4$. This implies that $M_{D}$ is the biggest subgraph of $G$ of maximal valency $\Delta=7$ and diameter $D$.

Theorem 4. Let $D$ be an odd natural number, and let $G$ be the infinite enhanced mesh network. Then,

$$
\left\{\begin{array}{l}
N_{G}(7,1)=3, \\
N_{G}(7, D)=\left|V\left(L_{D}\right)\right|, \quad \text { for } D=2 k+1, k \in \mathbb{N} .
\end{array}\right.
$$


Proof. For $D=1$, the graph contains $K_{3}$ and this is a biggest induced subgraph of $G$ of maximal valency 7 . Hence, $N_{G}(7,1)=3$.

For $D=2 k+1, k \in \mathbb{N}$, and $\Delta=7$, we construct a subgraph $Q_{D}$ (shown in Figure 5) from $L_{D}$ as follows.

Let $L$ be the horizontal line passing through edge $b c$ of the central triangle $T$ of graph $L_{D}$ and $M$ be the upper neighboring horizontal row. Let $K$ be the wheel subgraph of $L_{D}$ containing the central triangle $T$ :

(i) Delete the upper-left and upper-right hub edges in each $W_{5}$ lying on and above the line $M$ of $L_{D}$

(ii) Delete the lower-left and lower-right hub edges in each $W_{5}$ lying on and below the line $L$ of $L_{D}$

(iii) Delete the upper-left, lower-left hub edges in each $W_{5}$ lying on the left of wheel graph $K$.

(iv) Delete the upper-right and lower-right hub edges in each $W_{5}$ lying on the right of wheel graph $K$

(v) Furthermore, delete the left and right vertical edges in the wheel graph $K$

The resulting subgraph is denoted by $Q_{D}$ (shown in Figure 5 for $D=5,7,9,11$ ) is the spanning subgraph of $L_{D}$; hence, $V\left(L_{D}\right)=V\left(Q_{D}\right)$. Furthermore, the graph $Q_{D}$ also has diameter $D$ since the distance of all the nodes of $Q_{D}$ from the central triangle is the same as in $L_{D}$. This implies that $Q_{D}$ is the biggest subgraph of $G$ of maximal valency $\Delta=7$ and diameter $D$.

\subsection{Values for $\Delta=6$}

Theorem 5. Let $D$ be an even natural number, and let $G$ be the infinite enhanced mesh network. Then,

$$
\begin{cases}N_{G}(6, D)=7, & \text { for } D=2, \\ N_{G}(6, D)=\left|V\left(L_{D}\right)\right|, & \text { for } D=2 k, k \in \mathbb{N}-\{1\} .\end{cases}
$$

Proof. For $D=2$, the graph contains $K_{1,6}$. This is a biggest subgraph of $G$ of maximal valency 6 since in the graph $L_{D}$ (shown in Figure 3(a)) of maximal valency $\Delta=8$, and the central node can be connected to at most 6 nodes. Hence, $N_{G}(6,2)=7$.

For $D=2 k, k \in\{2,3,4, \ldots\}, \Delta=6$, we construct a subgraph $M_{D}$ (shown in Figure 6) from $L_{D}$ as follows.

Let $L$ and $M$ be the horizontal and vertical lines passing through the central node $n$ that divide $L_{D}$ into four regions (upper left, upper right, lower left, and lower right). Let $H$ and $K$ be the wheel graphs $W_{5}$ of $L_{D}$ lying in the upper-right and lower-left regions at a distance 0 from the central node $n$. Furthermore, suppose that $x$ and $y$ be the hub vertices of $H$ and $K$, respectively:

(i) Delete the upper-left, upper-right, and lower-left hub edges in each $W_{5}$ lying in the upper-left region of $L_{D}$

(ii) Delete the upper-left, upper-right, and lower-right hub edges in each $W_{5}$ lying in the upper-right region of $L_{D}$ except the wheel graph $H$ (iii) Delete the lower-left, lower-right, and upper-left hub edges in each $W_{5}$ lying in the lower-left region of $L_{D}$ except the wheel graph $K$

(iv) Delete the lower-left, lower-right, and upper-right hub edges in each $W_{5}$ lying in the lower-right region of $L_{D}$

(v) Furthermore, delete the upper-left, upper-right, lower-left hub edges, and right vertical edge in $H$; also, delete the lower-left, lower-right, upper-right hub edges, and left vertical edge in $K$

The resulting subgraph is denoted by $M_{D}$ (shown in Figure 6 , for $D=4,6,8,10)$, which is the spanning subgraph of $L_{D}$; hence, $V\left(L_{D}\right)=V\left(M_{D}\right)$. Furthermore, the graph $M_{D}$ also has diameter $D$ since the distance of all the nodes of $M_{D}$ from $n$ is the same as in $L_{D}$ except the nodes $x$ and $y$. However, $d(x, n)=2 \leq(D / 2), d(y, n)=2 \leq(D / 2)$, for all $D \geq 4$. This implies that $M_{D}$ is the biggest subgraph of $G$ of maximal valency $\Delta=6$ and diameter $D$.

Theorem 6. Let $D$ be an odd natural number, and let $G$ be the infinite enhanced mesh network. Then,

$$
\left\{\begin{array}{l}
N_{G}(6,1)=3 \\
N_{G}(6,3)=13 \\
N_{G}(6, D)=\left|V\left(L_{D}\right)\right|, \quad \text { for } D=2 k+1, k \in \mathbb{N} .
\end{array}\right.
$$

Proof. For $D=1$, the graph contains $K_{3}$ and this is a biggest induced subgraph of $G$ of maximal valency 6. Hence, $N_{G}(6,1)=3$.

For $D=3$, the graph $L_{D}$ of maximal valency 8 is a closed ball of radius 3 (shown Figure $3(\mathrm{~b})$ ). For maximal valency $\Delta=6$, the biggest subgraph cannot contain all the nodes of the graph $L_{D}$, otherwise $D=4$. Thus, the graph shown in Figure $7(\mathrm{a})$ is the biggest subgraph of maximal valency $\Delta=6$. Hence, $N_{G}(6,3)=13$.

For $D=2 k+1, k \in \mathbb{N}$, and $\Delta=6$, we construct a subgraph $Q_{D}$ (shown in Figure 7) from $L_{D}$ as follows.

Let $S$ be the wheel graph $W_{5}$ that contains the central triangle $T$. Let $R$ be the horizontal row of the wheel graphs $W_{5}$ containing the wheel graph $S$. Let $S_{1}\left(S_{2}\right)$ be the wheel graphs lying on left (right) of $S$ and is adjacent to it. Also, let $S_{3}$ be the wheel graph $W_{5}$ below the wheel graph $S$ and is adjacent to it:

(i) Delete the upper-left and upper-right hub edges in each $W_{5}$ lying above the row $R$ of $L_{D}$

(ii) Delete the lower-left and lower-right hub edges in each $W_{5}$ lying below the row $R$ of $L_{D}$

(iii) Delete the upper-left and lower-left hub edges in each $W_{5}$ lying on the row $R$ and left of wheel graph $S$

(iv) Delete the upper-right and lower-right hub edges in each $W_{5}$ lying on the line $R$ and right of wheel graph $S$

(v) Delete the vertical edges in each $W_{5}$ lying on the row $R$

(vi) Furthermore, delete the upper-right edge of $S_{1}$, lower-left edge of $S_{2}$, and upper-left edge of the $S_{3}$ 

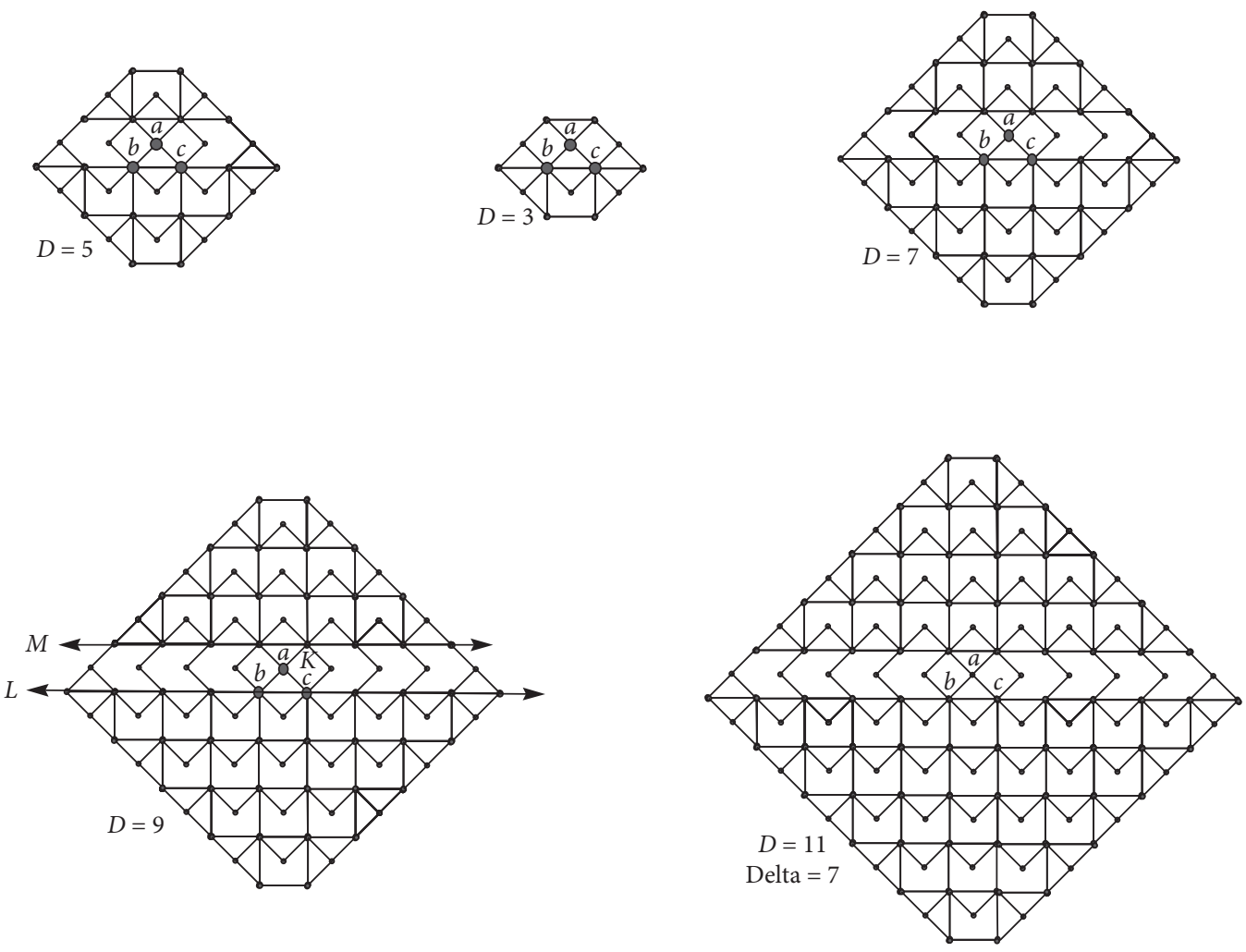

FIgURE 5: The biggest subgraphs of $G$ for $D=3,5,7,9,11$ and $\Delta=7$.
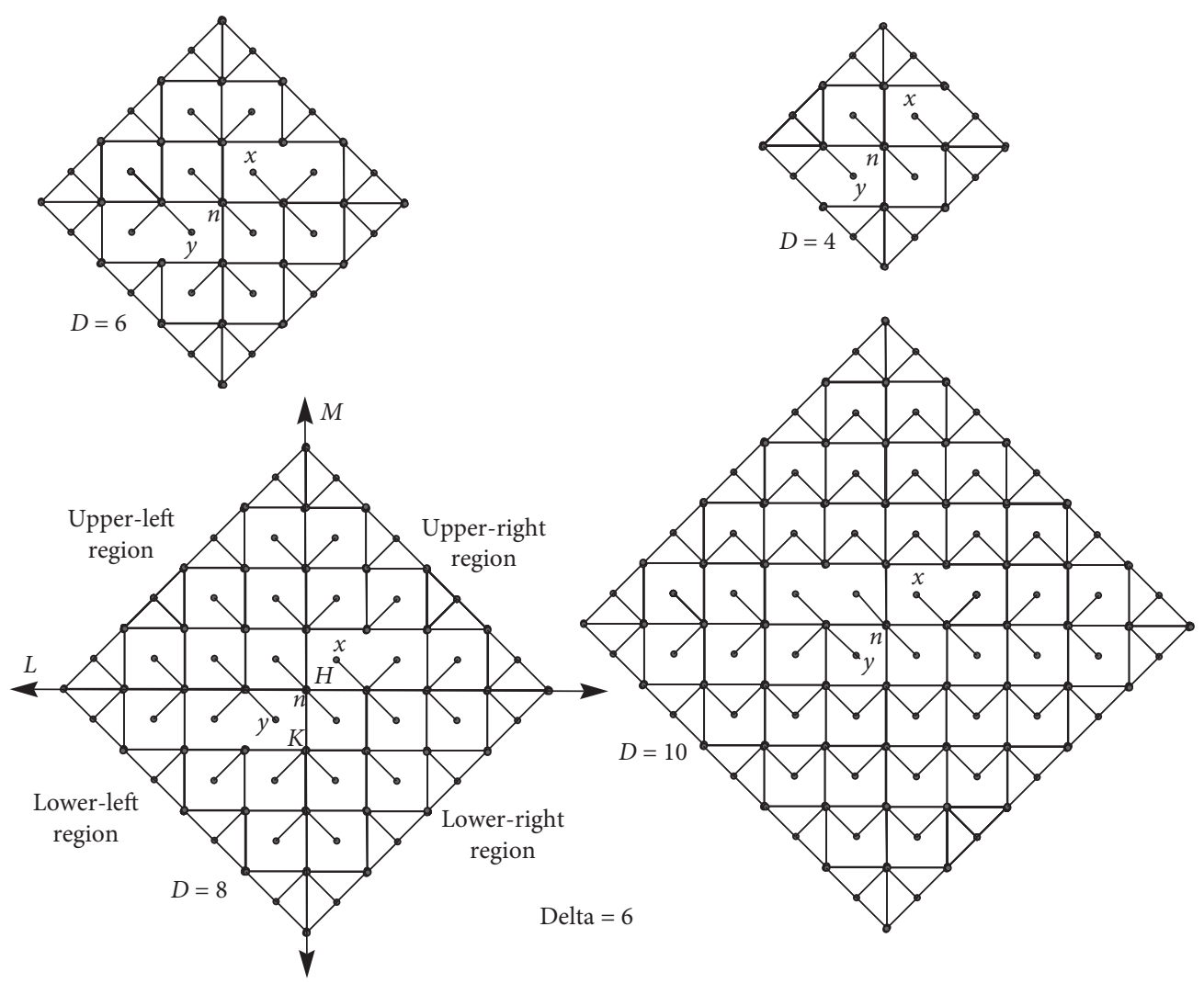

Figure 6: $M_{D}$ for $D=4,6,8,10$ and maximal valency $\Delta=6$. 

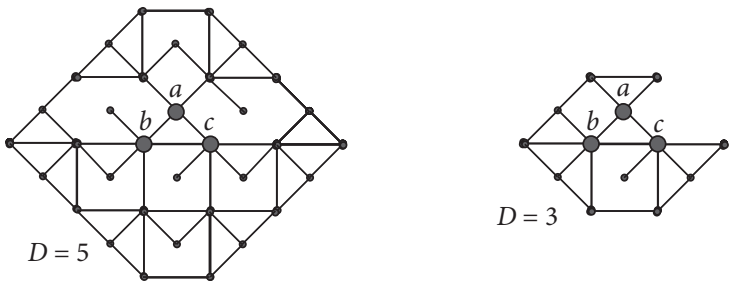

(a)

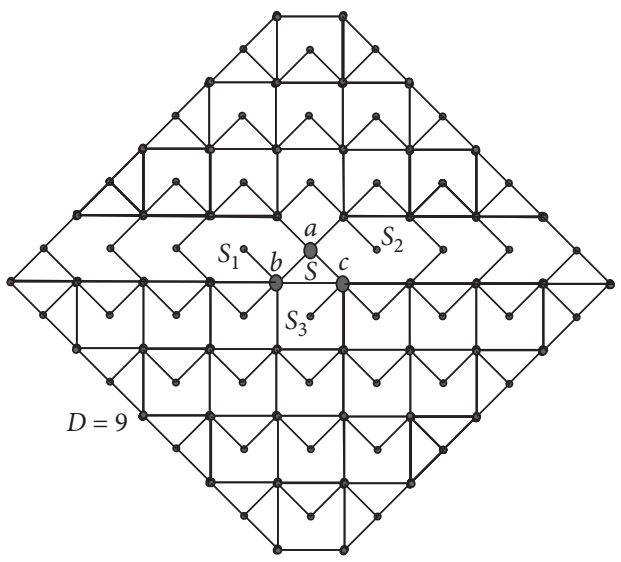

(b)
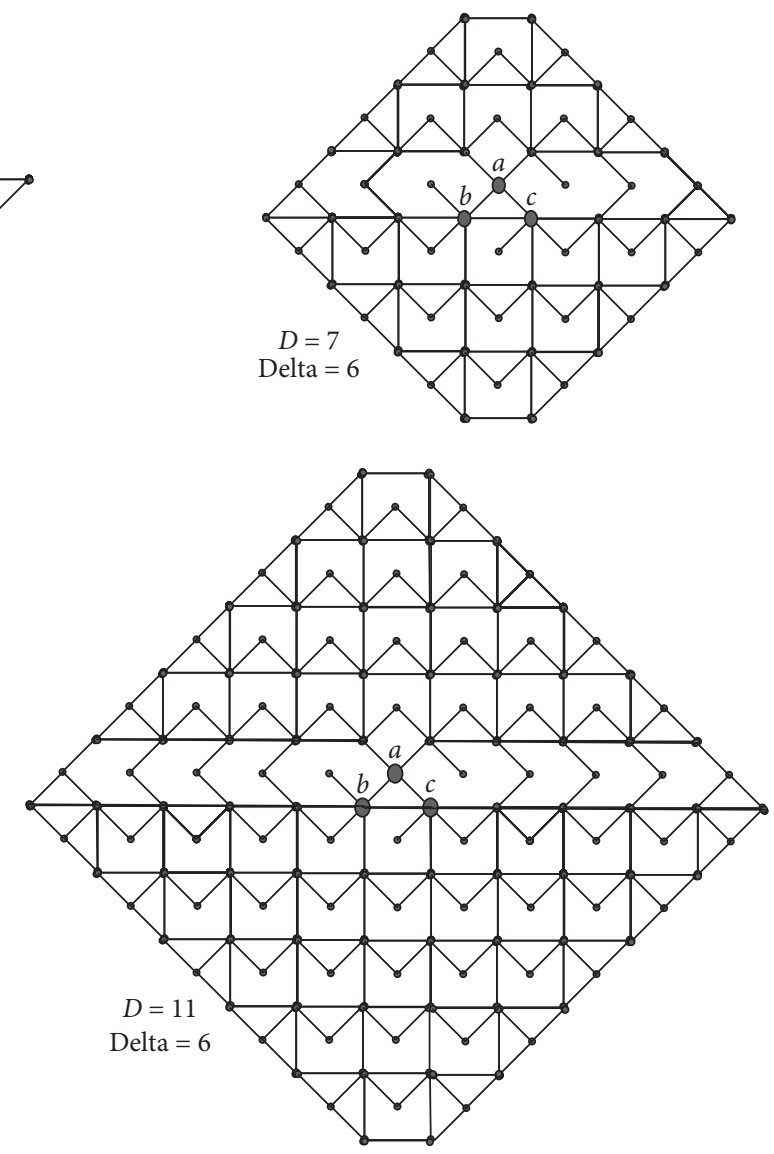

Figure 7: The biggest subgraphs of $G$ for $D=3,5,7,9,11$ and $\Delta=6$.

The resulting subgraph is denoted by $Q_{D}$ (shown in Figure 7 , for $D=5,7,9,11$ ), which is the spanning subgraph of $L_{D}$; hence, $V\left(L_{D}\right)=V\left(Q_{D}\right)$. Furthermore, the graph $Q_{D}$ also has diameter $D$ since the distance of all the nodes of $Q_{D}$ from the central triangle is the same as in $L_{D}$. This implies that $Q_{D}$ is the biggest subgraph of $G$ of maximal valency $\Delta=6$ and diameter $D$.

\subsection{Values for $\Delta=5$}

Theorem 7. Let $D$ be an even natural number, and let $G$ be the infinite enhanced mesh network. Then,

$$
\begin{cases}N_{G}(5, D)=6, & \text { for } D=2, \\ N_{G}(5, D) \geq 22, & \text { for } D=4, \\ N_{G}(5, D)=\left|V\left(L_{D}\right)\right|, & \text { for } D=2 k, k \in \mathbb{N}-\{1,2\}\end{cases}
$$

Proof. For $D=2$, the graph contains $K_{1,5}$. This is the biggest subgraph of $G$ of maximal valency 5 , since in graph $L_{D}$ (shown in Figure 3(a)) of maximal valency $\Delta=8$, the central node can be connected to at most 5 nodes. Hence, $N_{G}(5,2)=6$.
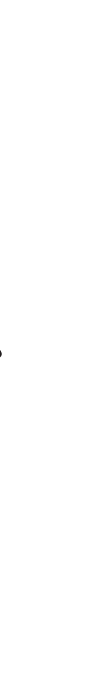


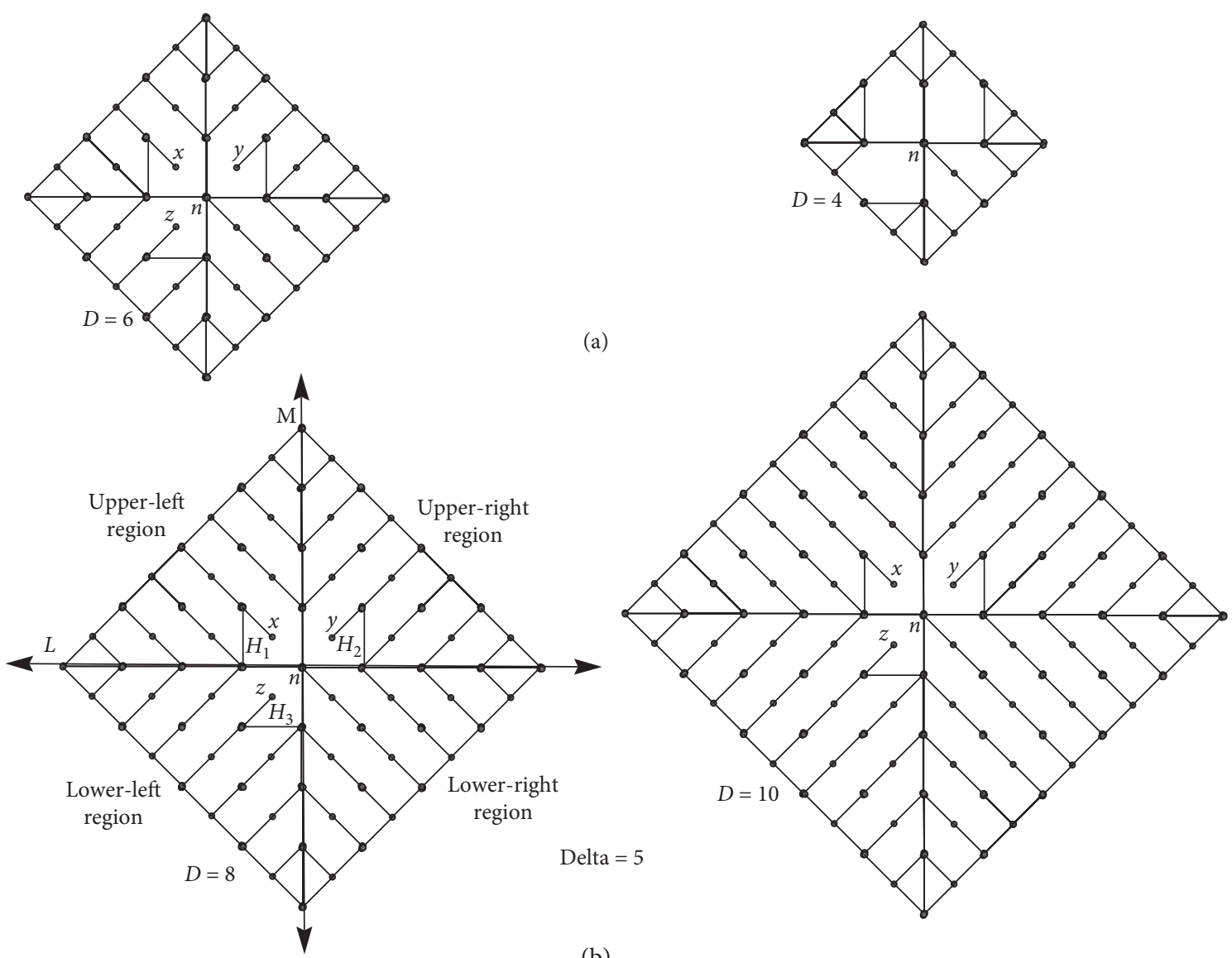

(b)

Figure 8: $M_{D}$ for $D=4,6,8,10$ and maximal valency $\Delta=5$.

(vii) Delete the three oblique edges of $H_{1}, H_{2}$, and $H_{3}$ that are adjacent to the central node $n$

(viii) Now reinstate the left vertical edge of $H_{1}$, right vertical edge of $\mathrm{H}_{2}$, and lower horizontal edge of $\mathrm{H}_{3}$

The resulting subgraph is denoted by $M_{D}$ (shown in Figure 7 , for $D=6,8,10)$, which is the spanning subgraph of $L_{D}$; hence, $V\left(L_{D}\right)=V\left(M_{D}\right)$. Furthermore, the graph $M_{D}$ also has diameter $D$ since the distance of all the nodes of $M_{D}$ from $n$ is the same as in $L_{D}$ except the nodes $x, y$, and $z$. However, $d(x, n)=2 \leq(D / 2), d(y, n)=2 \leq(D / 2)$ and $d(z, n)=2 \leq$ $(D / 2)$, for all $D \geq 6$. This implies that $M_{D}$ is the biggest subgraph of $G$ of maximal valency $\Delta=5$ and diameter $D$.

Theorem 8. Let $D$ be an odd natural number, and let $G$ be the infinite enhanced mesh network. Then,

$$
\left\{\begin{array}{l}
N_{G}(5,1)=3, \\
N_{G}(5,3) \geq 11, \\
N_{G}(5,5) \geq 33, \\
N_{G}(5, D)=\left|V\left(L_{D}\right)\right|, \quad \text { for } D=2 k+1, k \in \mathbb{N}-\{1,2\} .
\end{array}\right.
$$

Proof. For $D=1$, the graph contains $K_{3}$ and this is a biggest induced subgraph of $G$ of maximal valency 5. Hence, $N_{G}(5,1)=3$.
For $D=3$, the graph shown in Figure 9(a) is of maximal valency $\Delta=5$ and diameter 3 , which is a subgraph of $L_{D}$ (shown in Figure $3(\mathrm{~b})$ ). Hence, $N_{G}(5,3) \geq 11$.

For $D=5$, the graph shown in Figure 9(a) is of maximal valency $\Delta=5$ and diameter 5 , which is a subgraph of $L_{D}$ (shown in Figure $3(\mathrm{~b})$ ). Hence, $N_{G}(5,3) \geq 33$.

For $D=2 k+1, k \in \mathbb{N}-\{1,2\}$, and $\Delta=5$, we construct a subgraph $Q_{D}$ (shown in Figure 9) from $L_{D}$ as follows.

Let $L$ be the horizontal line passing through the edge $b c$ of the central triangle $T$ and $R$ be the vertical column of wheel graphs $W_{5}$ containing the central triangle $T$ that divide the graph $L_{D}$ into four regions (upper left, upper right, lower left, and lower right), as shown in Figure 9(b). Let $K$ be the wheel graph $W_{5}$ that contains the central triangle $T$. Let $K_{1}$ and $K_{3}$ be the wheel graphs $W_{5}$ lying in the lower-left and lower-right regions, respectively, at a distance zero from the central triangle $T$, both lying on the line $L$. Let $K_{2}$ be the wheel graph $W_{5}$ lying below the wheel graph $K$ and is adjacent to it:

(i) Delete the upper-right and lower-left hub edges in each $W_{5}$ lying in the upper-left region of $L_{D}$

(ii) Delete the upper-right and lower-left hub edges in each $W_{5}$ lying in the lower-right region of $L_{D}$

(iii) Delete the upper-left and lower-right hub edges in each $W_{5}$ lying in the upper-right region of $L_{D}$ 

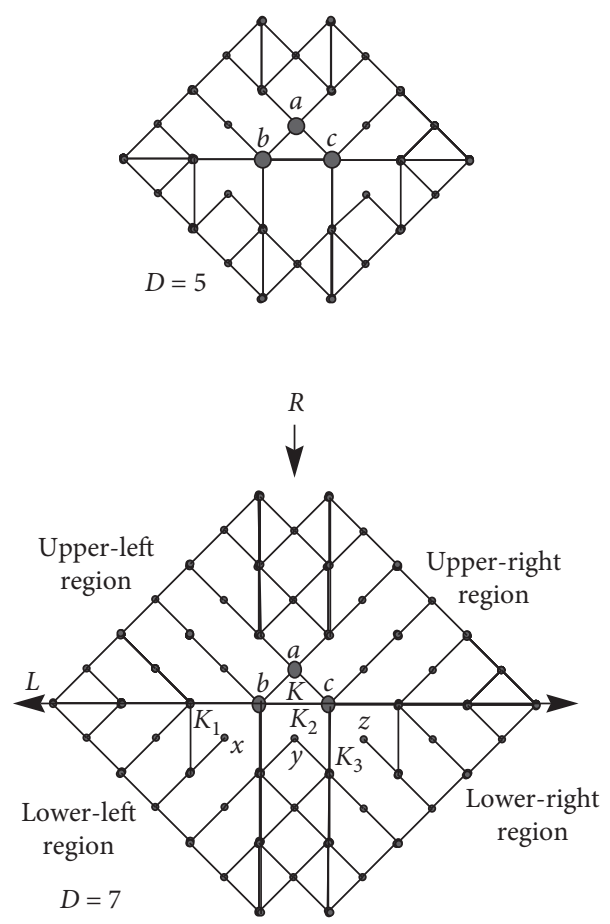

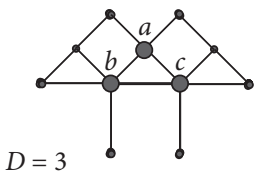

(a)

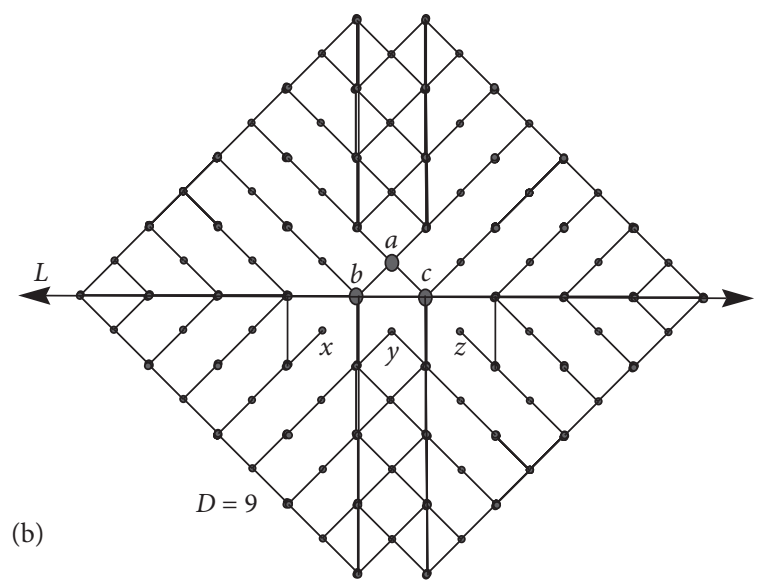

FIgURE 9: The biggest subgraphs of $G$ for $D=3,5,7,9$ and $\Delta=5$.

(iv) Delete the upper-left and lower-right hub edges in each $W_{5}$ lying in the lower-left region of $L_{D}$

(v) Delete all the horizontal edges of $L_{D}$ in the four regions except the edges that are lying on the horizontal line $L$

(vi) Delete all the vertical edges of $L_{D}$ in the four regions except the edges that are lying on the vertical column $R$

(vii) Delete the left and right vertical edges of wheel graph $K$

(viii) Delete the upper-left and upper-right hub edges in the wheel graph $K_{2}$

(ix) Delete the upper-right (upper-left) hub edge of the wheel graph $K_{1}\left(K_{3}\right)$

(x) Now, reinstate the left vertical edge of $K_{1}$ and right vertical edge of $K_{3}$

The resulting subgraph is denoted by $Q_{D}$ (shown in Figure 9, for $D=7,9)$, which is the spanning subgraph of $L_{D}$; hence, $V\left(L_{D}\right)=V\left(Q_{D}\right)$. Furthermore, the graph $Q_{D}$ also has diameter $D$ since the distance of all the nodes of $Q_{D}$ from the central triangle is the same as in $L_{D}$ except the nodes $x, y$, and $z$ whose distance from central triangle $T$ is $\leq((D-1) / 2)$. This implies that $Q_{D}$ is the biggest subgraph of $G$ of maximal valency $\Delta=5$ and diameter $D$.

4.5. Values and Bounds for $\Delta=4$. Now, we discuss the case when $\Delta=4$.

For even $D$, we prove the succeeding theorem.
Theorem 9. Let $G$ be the infinite enhanced mesh and $D$ be an even natural number. Then,

$$
\begin{cases}N_{G}(4, D)=5, & \text { for } D=2, \\ N_{G}(4, D) \geq 17, & \text { for } D=4, \\ N_{G}(4, D) \geq 41, & \text { for } D=6, \\ N_{G}(4, D) \geq\left|V\left(L_{D}\right)\right|-4, & \text { for } D=2 k, k \in \mathbb{N}-\{1,2,3\} .\end{cases}
$$

Proof. The lower bound for $N_{G}(4,2)$ is 5 , since the graph contains $K_{1,4}$. The biggest subgraph of $G$ of maximal valency 8 is the graph $L_{D}$ (shown Figure $3(\mathrm{a})$ ). When $\Delta=4$, the central node $n$ of $L_{D}$ can be connected to at most four vertices. Therefore, we have to remove at least four vertices, which are adjacent to $n$, otherwise $D>2$. This implies that the biggest subgraph of $G$ of maximal valency 4 can contain at most 5 vertices. Hence, $N_{G}(4,2)=5$.

For $D=4$, the graph $G$ contains the subgraph (shown in Figure $10(\mathrm{a})$ ) that has maximal valency and diameter 4 . Thus, $N_{G}(4, D) \geq 17$.

For $D=6$, the graph $G$ contain the subgraph (shown in Figure 10(a)) that has maximal valency 4 and diameter 6 . Thus, $N_{G}(4, D) \geq 41$.

For $D=2 k, k \in\{3,4, \ldots\}$, and $\Delta=4$, we construct a subgraph $M_{D}$ (shown in Figure 10) from $L_{D}$ as follows.

Let $L$ and $M$ be the horizontal and vertical lines, respectively, passing through the central node $n$ that divide $L_{D}$ into four regions (upper left, upper right, lower left, and lower right). Let $H_{1}, H_{2}, H_{3}$, and $H_{4}$ be the wheel graphs $W_{5}$ of $L_{D}$ lying in the upper-left, upper-right, lower-left, and 

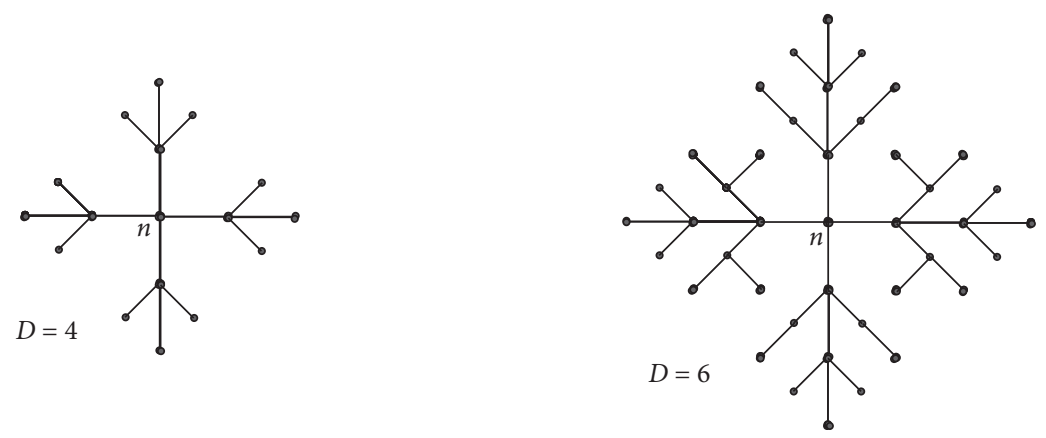

(a)
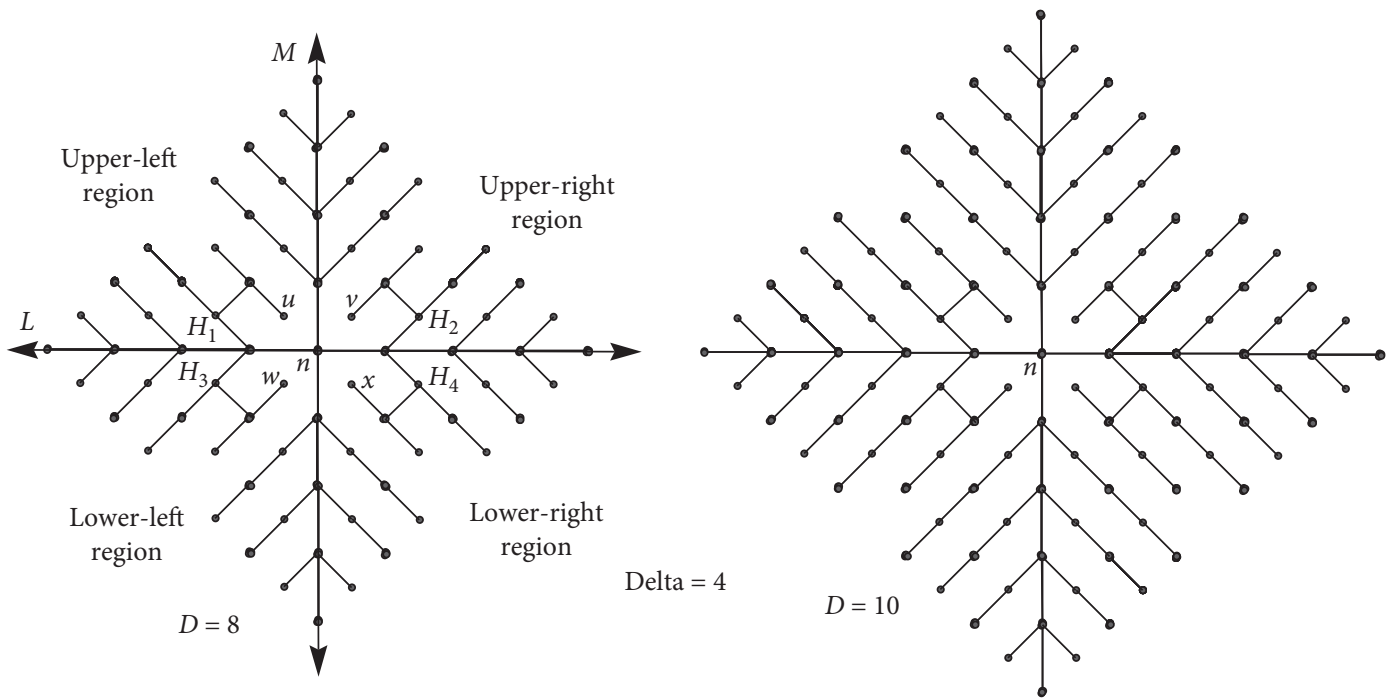

(b)

Figure 10: The subgraphs $M_{D}$ of $G$ for $D=4,6,8,10$ and $\Delta=4$.

lower-right regions, respectively, at a distance 1 from the central node $n$ and lying on the line $L$. Let $P_{1}$ be the path of length $D$ passing through the central node $n$ and lies in upper-left and lower-right regions. Let $P_{2}$ be the path of length $D$ passing through the central node $n$ and lies in upper-right and lower-left regions. Furthermore, suppose that the nodes $u$ and $v$ lie on the path $P_{1}$ and $w$ and $x$ lie on the path $P_{2}$ that are adjacent to the central node $n$ :

(i) Delete the upper-right and lower-left hub edges in each $W_{5}$ lying in the upper-left region of $L_{D}$

(ii) Delete the upper-right and lower-left hub edges in each $W_{5}$ lying in the lower-right region of $L_{D}$

(iii) Delete the upper-left and lower-right hub edges in each $W_{5}$ lying in the upper-right region of $L_{D}$

(iv) Delete the upper-left and lower-right hub edges in each $W_{5}$ lying in the lower-left region of $L_{D}$

(v) Delete all the horizontal edges in $L_{D}$ except the edges lying on the line $L$

(vi) Delete all the vertical edges in $L_{D}$ except the edges lying on the line $M$

(vii) Delete the four oblique edges $u n, v n, w n$, and $x n$ that are adjacent to the central node $n$ (viii) Delete both the end vertices of paths $P_{1}$ and $P_{2}$

(ix) Now, reinstate the upper-right hub edge of $H_{1}$, upper-left hub edge of $\mathrm{H}_{2}$, lower-right hub edge of $\mathrm{H}_{3}$, and lower-left hub edge of $\mathrm{H}_{4}$

The resulting subgraph is denoted by $M_{D}$ (shown in Figure 10 for $D=8,10)$. Furthermore, the graph $M_{D}$ has also diameter $D$ since the distance of all the nodes of $M_{D}$ from $n$ is the same as in $L_{D}$ except the nodes $u, v, w$, and $x$ whose distance from $n$ is $\leq(D / 2)$ for all $D \geq 8$. Since $M_{D}$ is obtained from $L_{D}$ by deleting only four nodes of $L_{D}$, which implies that $\left|V\left(M_{D}\right)\right|=\left|V\left(L_{D}\right)\right|-4$.

Hence, $N_{G}(4, D) \geq\left|V\left(L_{D}\right)\right|-4$.

Theorem 10. Let $D$ be an odd natural number, and let $G$ be the infinite enhanced mesh network. Then,

$$
\left\{\begin{array}{l}
N_{G}(4,1)=3, \\
N_{G}(4,3) \geq 9, \\
N_{G}(4,5) \geq 27, \\
N_{G}(4,7) \geq 59, \\
N_{G}(4, D)=\left|V\left(L_{D}\right)\right|, \quad \text { for } D=2 k+1, k \in \mathbb{N}-\{1,2,3\} .
\end{array}\right.
$$


Proof. For $D=1$, the graph contains $K_{3}$ and this is a biggest induced subgraph of $G$ of maximal valency 4. Hence, $N_{G}(4,1)=3$.

For $D=3$, the graph shown in Figure 11(a) is of maximal valency $\Delta=4$ and diameter 4 , which is a subgraph of $L_{D}$ (shown in Figure 3(b)). Hence, $N_{G}(4,3) \geq 9$.

For $D=5$, the graph shown in Figure 11(a) is of maximal valency $\Delta=4$ and diameter 5 , which is a subgraph of $L_{D}$ (shown in Figure 3(b)). Hence, $N_{G}(4,5) \geq 27$.

For $D=7$, the graph shown in Figure $11(\mathrm{~b})$ is of maximal valency $\Delta=4$ and diameter 7 , which is a subgraph of $L_{D}$ (shown in Figure $3(\mathrm{~b})$ ). Hence, $N_{G}(4,7) \geq 59$.

For $D=2 k+1, k \in \mathbb{N}-\{1,2,3\}$, and $\Delta=4$, we construct a subgraph $Q_{D}$ (shown in Figure 11) from $L_{D}$ as follows

Let $L$ be the horizontal line passing through the edge $b c$ of the central triangle $T$ and $R$ be the vertical column of wheel graphs $W_{5}$ containing the central triangle $T$ that divide the graph $L_{D}$ into four regions (upper left, upper right, lower left, and lower right). Let $K$ be the wheel graph $W_{5}$ that contains the central triangle $T$. Let $K_{1}$ and $K_{3}$ be the wheel graphs $W_{5}$ lying in the lower-left and lowerright regions, respectively, at a distance 1 from the central triangle $T$, both lying on the line $L$. Let $K_{2}$ be the wheel graph $W_{5}$ lying below the wheel graph $K$ and is adjacent to it. Let $K_{4}\left(K_{6}\right)$ be the wheel graph $W_{5}$ lying on left (right) of the wheel graph $K$ and is adjacent to it. Let $K_{5}$ be the wheel graph $W_{5}$ lying above the wheel graph $K$ and is adjacent to it:

(i) Delete upper-right and lower-left hub edges in each $W_{5}$ lying in the upper-left region of $L_{D}$

(ii) Delete the upper-right and lower-left hub edges in each $W_{5}$ lying in the lower-right region of $L_{D}$

(iii) Delete the upper-left and lower-right hub edges in each $W_{5}$ lying in the upper-right region of $L_{D}$

(iv) Delete the upper-left and lower-right hub edges in each $W_{5}$ lying in the lower-left region of $L_{D}$

(v) Delete all the horizontal edges of $L_{D}$ in the four regions except the edges that are lying on the line $L$

(vi) Delete all the vertical edges of $L_{D}$ in the four regions except the edges that are lying in the column $R$

(vii) Delete the left and right vertical edges of $K$

(viii) Delete the upper-left, upper-right, and lower-right hub edges in each $W_{5}$ lying in the column $R$ and above $K$ except $K_{5}$

(ix) Delete the lower-left, lower-right, and upper-left hub edges in each $W_{5}$ lying in the column $R$ and below $K$ except $K_{2}$

(x) Delete the upper-left, upper-right, and lower-right hub edges in $K_{2}$

(xi) Delete the lower-left, lower-right, and upper-left hub edges in $K_{5}$

(xii) Furthermore, delete the edges $y b, w b, t c$, and $u c$

(xiii) Now, reinstate the lower horizontal edges in $K_{1}$ and $K_{3}$ (xiv) Now, reinstate the upper horizontal edges in $K_{4}$ and $K_{6}$

The resulting subgraph is denoted by $Q_{D}$ (shown in Figure 11(b), for $D=9$ ), which is the spanning subgraph of $L_{D}$; hence, $V\left(L_{D}\right)=V\left(Q_{D}\right)$. Furthermore, the graph $Q_{D}$ also has diameter $D$ since the distance of all the nodes of $Q_{D}$ from the central triangle is the same as in $L_{D}$ except the nodes $s, t$, $u, v, w, x, y$, and $z$ whose distance from central triangle $T$ is $\leq((D-1) / 2)$. This implies that $Q_{D}$ is the biggest subgraph of $G$ of maximal valency $\Delta=4$ and diameter $D$.

4.6. Bounds for $\Delta=3$. For $D=1$, the graph contains $K_{3}$ and this is a biggest induced subgraph of $G$ of maximal valency 3 . Hence, $N_{G}(3,1)=3$.

The lower bound for $N_{G}(3,2)$ is 4 , since the graph contains $K_{1,3}$. The biggest subgraph of $G$ of maximal valency 8 is the graph $L_{D}$ (shown in Figure 3(a)). When $\Delta=3$, the central node $n$ of $L_{D}$ can be connected to at most three nodes. Therefore, we have to remove at least three nodes which are adjacent to $n$, otherwise $D>2$. This implies that the biggest subgraph of $G$ of maximal valency 3 can contain at most 4 nodes. Hence, $N_{G}(3,2)=4$.

Theorem 11. Let $G$ be the infinite enhanced mesh network and $D$ be an even natural number. Then,

$$
N_{G}(3, D) \geq \begin{cases}10, & \text { for } D=4, \\ 22, & \text { for } D=6, \\ 46, & \text { for } D=8, \\ \left|V\left(L_{D}\right)\right|-(8 k+33), & \text { for } D=2 k+8, k \in \mathbb{N} .\end{cases}
$$

Proof. For $D=4,6,8$, the subgraphs of $G$ shown in Figure 12 are of maximal valency $\Delta=3$. It is also easy to check that the diameter of each graph is $D$. Hence, the theorem is true for $D=4,6,8$.

For $D=2 k+8, L_{D}$ is the biggest subgraph of $G$ of maximal valency $\Delta=8$ and diameter $D$. Let $W_{D}$ be the subgraph of $L_{D}$ of maximal valency $\Delta=3$ and diameter $D$. The structure of the graph $W_{D}$ for $D=10,12,14$ is depicted in Figure 12. The central node of graph $W_{D}$ correspond to the central node of $L_{D}$ and is exhibited by $\bullet$. The whole graph $W_{D}$ is constructed from $L_{D}$ by deleting those nodes which have distance greater than $(D / 2)$ from the central node $n$. The structure of the deleted nodes in the construction of $W_{D}$ is shown in Figure 12. To calculate the deleted nodes of $L_{D}$, we draw horizontal lines on deleted nodes and counting them in horizontal rows from top to bottom:

Delete vertices in $L_{D}=2+\{3+3+3+\cdots$ to $(k+1)$ terms $\}+4+(2 k+8)+2+5+4+\{3+3+3+\cdots$ to $(k+1)$ terms $\}+2=8 k+33$.

Hence, $\left|W_{D}\right|=\left|V\left(L_{D}\right)\right|-(8 k+33)$ for $D=2 k+8$.

Furthermore, $d(x, n) \leq(D / 2)$ for every node $x \in W_{D}$. Thus, $W_{D}$ has diameter at most $D$.

Hence, the theorem is satisfied for $D=2 k+8$, $k \in \mathbb{N}$. 

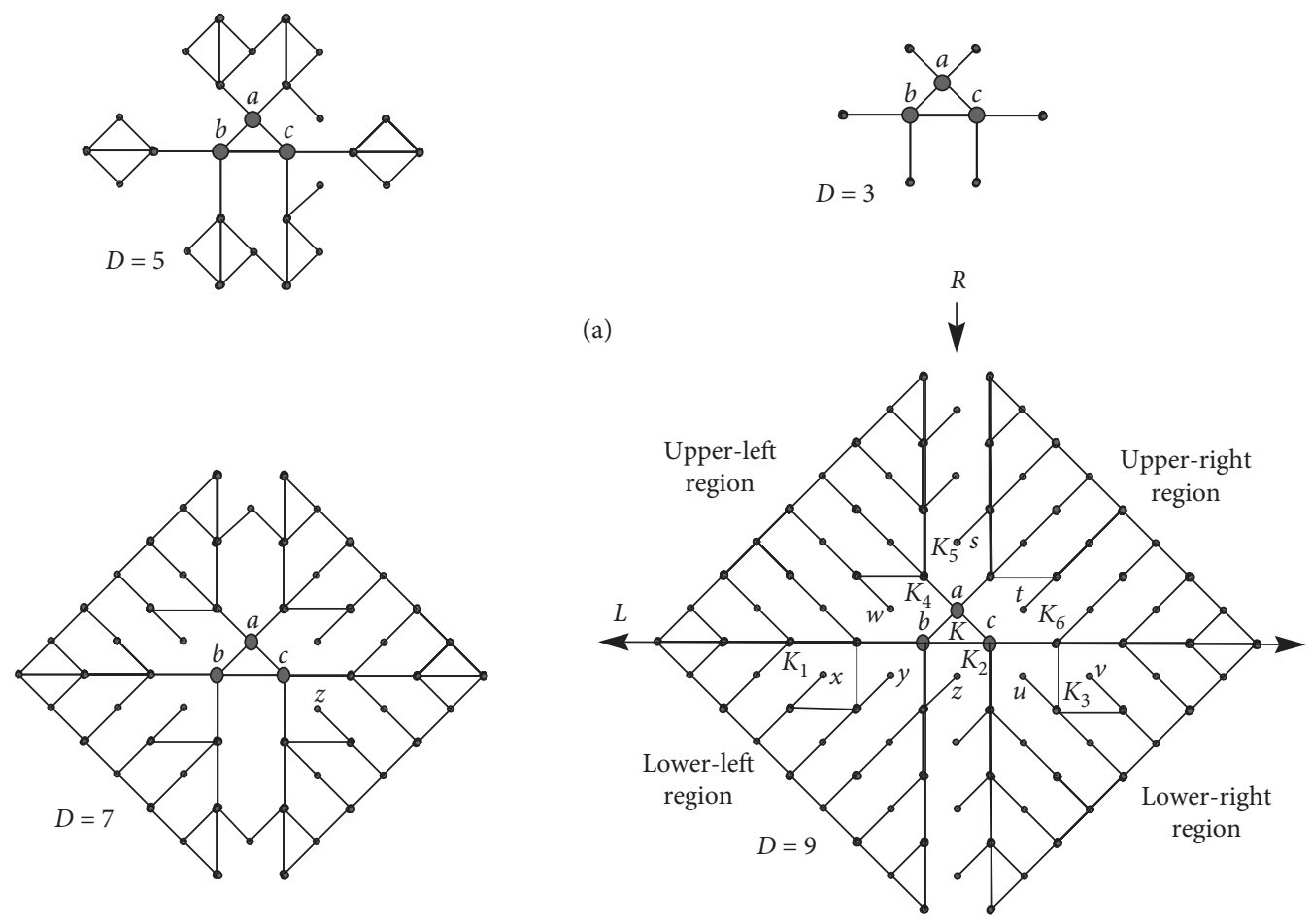

(b)

Figure 11: The biggest subgraphs of $G$ for $D=3,5,7,9$ and $\Delta=4$.
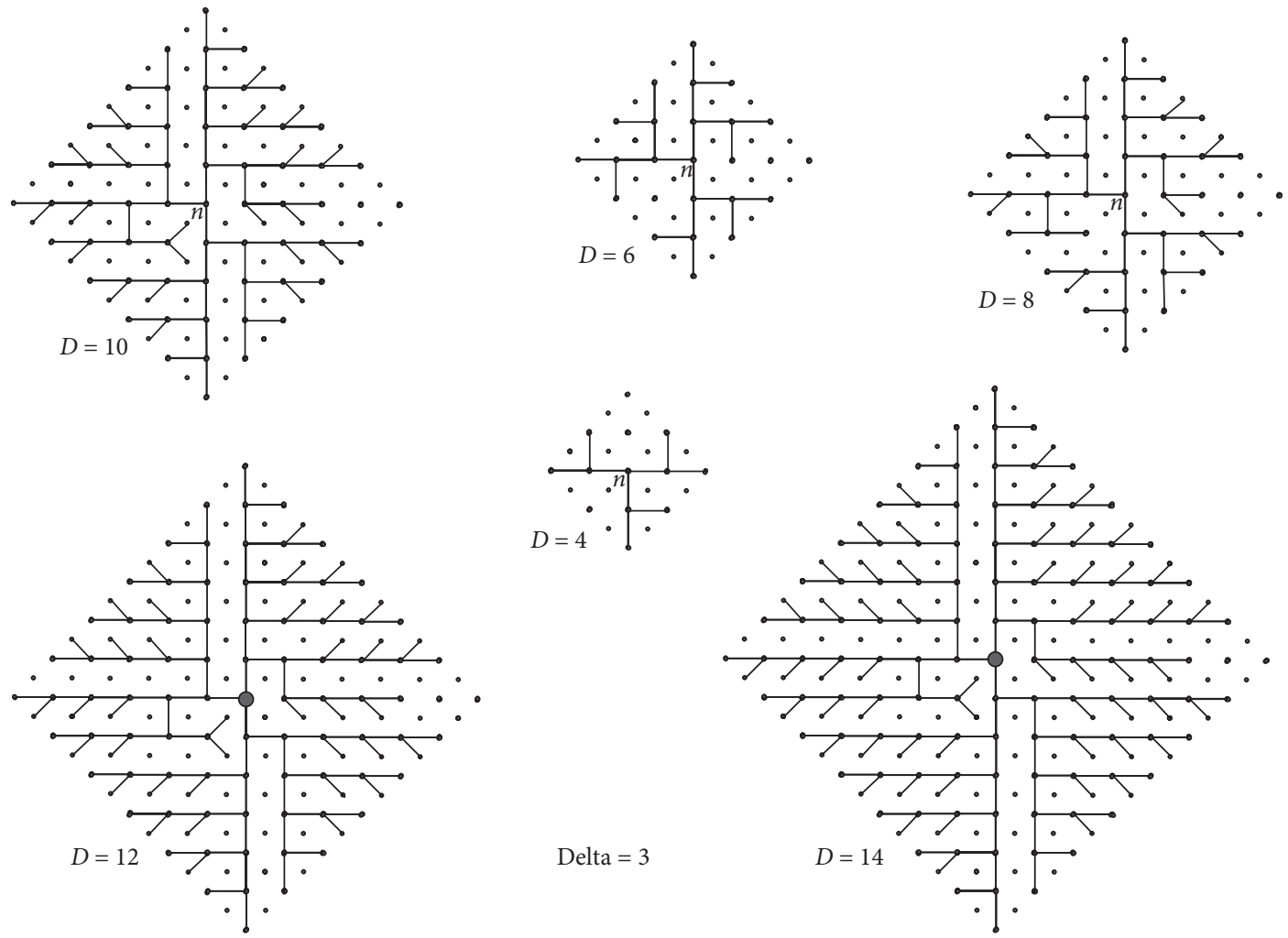

Figure 12: The subgraphs of $G$ for $D=4,6,8,10,12,14$ and $\Delta=3$. 


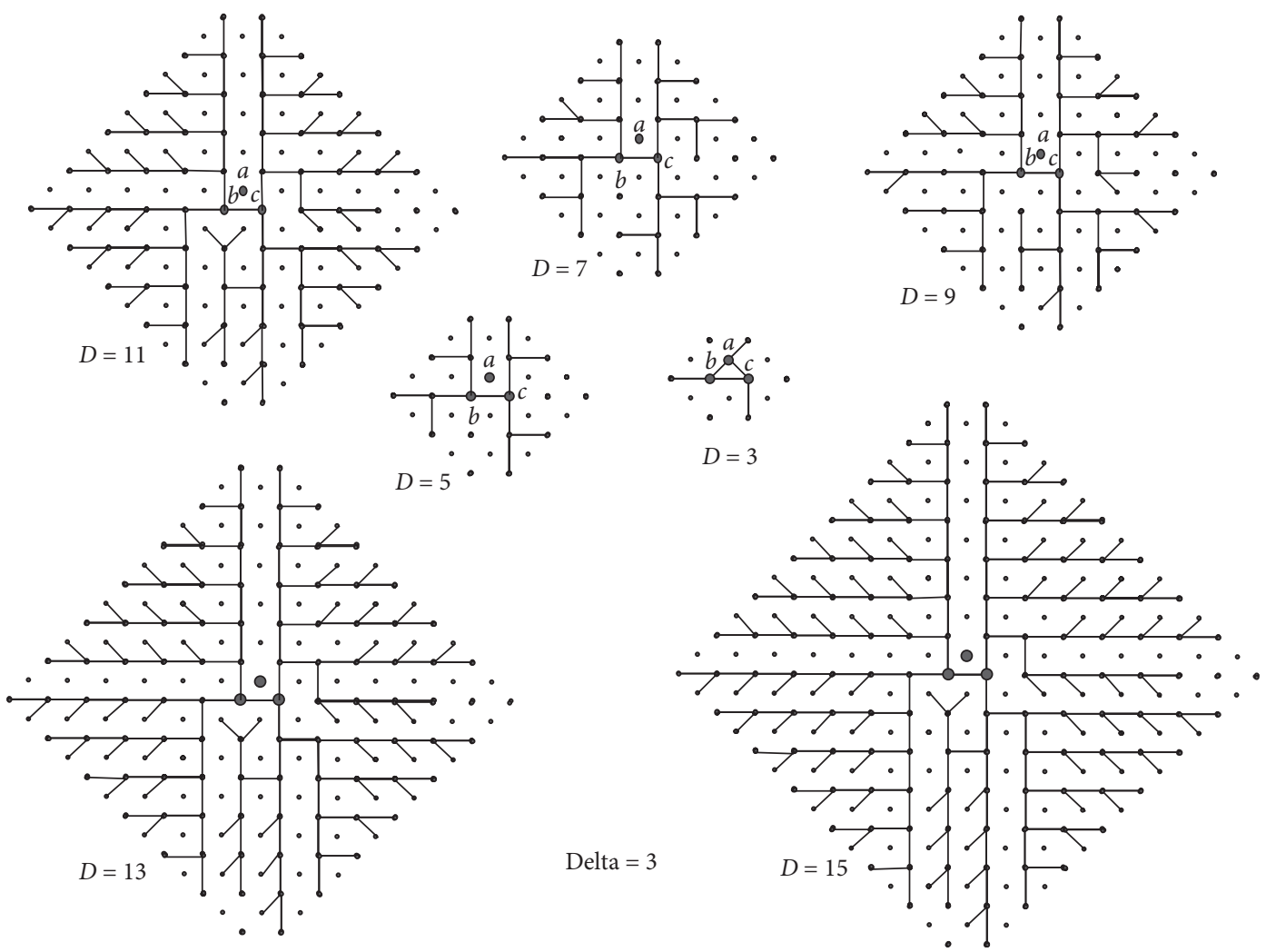

Figure 13: The subgraphs of $G$ for $D=3,5,7,9,11,13,15$ and $\Delta=3$.

Theorem 12. Let $G$ be the infinite enhanced mesh network and $D$ be an odd natural number. Then,

$$
N_{G}(3, D) \geq \begin{cases}6, & \text { for } D=3, \\ 14, & \text { for } D=5, \\ 29, & \text { for } D=7, \\ 56, & \text { for } D=9, \\ \left|V\left(L_{D}\right)\right|-(8 k+38), & \text { for } D=2 k+9 .\end{cases}
$$

Proof. For $D=3,5,7,9$, the subgraphs of $G$ shown in Figure 13 are of maximal valency $\Delta=3$. It is also easy to check that each graph has a diameter $D$. Hence, the theorem is true for $D=3,5,7,9$.

For $D=2 k+9, L_{D}$ is the biggest subgraph of $G$ of maximal valency $\Delta=8$ and diameter $D$. Let $W_{D}$ be the subgraph of $L_{D}$ of maximal valency $\Delta=3$ and diameter $D$. The structure of the graph $W_{D}$ for $D=11,13,15$ is exhibited in Figure 13. The central triangle of $W_{D}$ correspond to the central triangle $T$ of $L_{D}$ and is depicted by $\bullet$. The whole graph $W_{D}$ is constructed from $L_{D}$ by deleting those nodes which have distance greater than $((D-1) / 2)$ from the central triangle $T$. The structure of the deleted nodes in the construction of $W_{D}$ is shown in Figure 13. Now, calculate the deleted nodes of $L_{D}$ in horizontal rows from top to bottom.

Delete Vertices in $L_{D}=\{3+3+3+\cdots$ to $(k+2)$ terms $\}+$ $4+(2 k+9)+2+4+5+5+\{3+3+3+\cdots$ to $(k)$ terms $\}+$ $2+1=8 k+38$. $k \in \mathbb{N}$.

Hence, $\left|W_{D}\right|=\left|V\left(L_{D}\right)\right|-(8 k+38)$ for $D=2 k+9$,

Furthermore, $d(x, T) \leq((D-1) / 2)$ for every node $x \in W_{D}$. Thus, $W_{D}$ has diameter at most $D$.

Hence, the theorem is satisfied for $D=2 k+9, k \in \mathbb{N}$.

Using the result from Theorems 11 and 12 and the fact that $N_{G}(\Delta-1, D) \leq N_{G}(\Delta, D)$ for any graph, we get the succeeding statement.

Corollary 2. Let $G$ be the infinite enhanced mesh network and let $D \geq 10$ is a natural number. Then, for $k \in \mathbb{N}$,

$$
\begin{array}{ll}
\left|V\left(L_{D}\right)\right|-(8 k+33) \leq N_{G}(3, D) \leq\left|V\left(L_{D}\right)\right| & \text { for } D=2 k+8, \\
\left|V\left(L_{D}\right)\right|-(8 k+38) \leq N_{G}(3, D) \leq\left|V\left(L_{D}\right)\right| & \text { for } D=2 k+9 .
\end{array}
$$

\section{Conclusion}

In the Degree Diameter Problem, the largest graphs in term of vertices are computed with given degree and diameter. In this work, we have considered the restricted version of Degree Diameter Problem which states that given connected undirected host graph $G$, an upper bound $D$ for the maximum degree, and an upper bound $\Delta$ for the diameter find the largest connected subgraph of maximum degree $\leq \Delta$ and diameter $\leq D$. This problem becomes of particular interest when we consider the host graph as the network.

The topology of a network (such as a telecommunications, multiprocessor, or local area network, to name just a 
few) is usually modeled by a graph in which vertices represent 'nodes' (stations or processors) while undirected or directed edges stand for 'links' or other types of connections. There are many important features in the designing of such networks. One of the important aspects is to put limitation on vertex degree and its diameter. These two parameters in networks are interpreted as follows. By the degree of a node, it is meant to have the number of connections attached to that node; on the contrary, the diameter shows the largest number of links that must be required to transmit a message between any two nodes. The natural question that arises in this case is "what is then the largest number of nodes in a network with a limited degree and diameter?" If we design the network so that there is no directed edge, then this leads to the Degree/Diameter Problem.

Planar graphs are popular for network design because they have a convenient physical layout. Of all planar network topologies, the (square) mesh is the most popular and this has been studied in $[1,3-5,7]$. In this work, we have extended this study by considering the host graph $G$ as the graph obtained from the enhanced mesh network.

In future, it will be interesting to find more such mesh networks and study the optimal subgraphs.

Furthermore, we can extend this study to directed networks.

\section{Data Availability}

Data from previous studies were used to support this study. They are cited at relevant places within the text as references.

\section{Conflicts of Interest}

The authors declare that they have no conflicts of interest.

\section{Acknowledgments}

This research was supported by UPAR Grants of United Arab Emirates University (UAEU) under Grant nos. G00002590 and G00003271.

\section{References}

[1] M. Miller, H. Pérez-Rosés, and J. Ryan, "The maximum degree and diameter-bounded subgraph in the mesh," Discrete Applied Mathematics, vol. 160, no. 12, pp. 1782-1790, 2012.

[2] M. Miller and J. Sirán, "Moore graphs and beyond: a survey of the degree/diameter problem," The Electronic Journal of Combinatorics, vol. 1000, pp. 1-92, 2013.

[3] M. S. Akhtar, "Degree diameter problem on oxide networks," Journal of Computational and Applied Mathematics, vol. 7, pp. 1-7, 2018.

[4] M. S. Akhtar and S. A. Bokhary, "Degree diameter problem on silicate network," Utilitas Mathematica, 2018, In press.

[5] P. Holub, M. Miller, H. Pérez-Rosés, and J. Ryan, "Degree diameter problem on honeycomb networks," Discrete Applied Mathematics, vol. 179, pp. 139-151, 2014.

[6] P. Holub and J. Ryan, "Degree diameter problem on triangular networks," The Australasian Journal of Combinatorics, vol. 63, no. 3, pp. 333-345, 2015.
[7] A. Dekker, H. Pérez-Rosés, G. Pineda-Villavicencio, and P. Watters, "The maximum degree and diameter-bounded subgraph and its applications," Journal of Mathematical Modelling and Algorithms, vol. 11, no. 3, pp. 249-268, 2002.

[8] M. S. Akhtar, U. Ali, G. Abbas, and M. Batool, "On the game chromatic number of splitting graphs of path and cycle," Theoretical Computer Science, vol. 795, pp. 50-56, 2019.

[9] J.-B. Liu, J. Zhao, and Z. X. Zhu, "On the number of spanning trees and normalized Laplacian of linear octagonal quadrilateral networks," International Journal of Quantum Chemistry, vol. 119, p. 25971, 2019.

[10] J.-B. Liu, J. Zhao, J. Min, and J. D. Cao, "On the hosoya index of graphs formed by a fractal graph," Fractals-Complex Geometry Patterns and Scaling in Nature and Society, vol. 27, no. 3, Article ID 1950135, 2019.

[11] J.-B. Liu, J. Zhao, H. He, and Z. Shao, "Valency-based topological descriptors and structural property of the generalized sierpiński networks," Journal of Statistical Physics, vol. 177, no. 6, pp. 1131-1147, 2019.

[12] J. A. Bondy and U. S. R. Murty, Graph Theory, Springer, Berlin, Germany, 2008.

[13] G. Exoo, "A family of graphs and the degree/diameter problem," Journal of Graph Theory, vol. 37, no. 2, pp. 118-124, 2001.

[14] M. Garey, D. Johnson, Computers, and Intractability, A Guide to the Theory of NP-Completeness, Freeman and Co., New York, NY, USA, 1979.

[15] S. Hayat and M. Imran, "Computation of topological indices of certain networks," Applied Mathematics and Computation, vol. 240, pp. 213-228, 2014. 